\title{
QCD Sum Rules, Scattering Length and the Vector Mesons in Nuclear Medium
}

\author{
Tetsuo Hatsuda ${ }^{1,2}$, Su Houng Lee ${ }^{2,3}$, and Hiroyuki Shiomi ${ }^{1}$ \\ ${ }^{1}$ Institute of Physics, University of Tsukuba, Tsukuba, Ibaraki 305, Japan \\ ${ }^{2}$ Institute for Nuclear Theory, NK-12, Univ. of Washington, Seattle, WA 98195, USA \\ 3 Department of Physics, Yonsei University, Seoul 120-749, Korea
}

\begin{abstract}
Critical examination is made on the relation between the mass shift of vector mesons in nuclear medium and the vector-meson - nucleon scattering length. We give detailed comparison between the QCD sum rule approach by two of the present authors (Phys. Rev. C46 (1992) R34) and the scatteringlength approach by Koike (Phys. Rev. C51 (1995) 1488). It is shown that the latter approach is mortally flawed both technically and conceptually.
\end{abstract}

PACS numbers: 24.85.+p, 12.38.Lg, 21.65.+f 


\section{INTRODUCTION}

The question of changes in the vector meson properties in nuclear medium is of interest in relation to the physics of nuclear matter [1] and relativistic heavy ion collision [2]. In particular, if there are spectral changes of vector mesons in medium, it could be observed directly through the lepton-pair spectrum in $\gamma-A, p-A$ and $A-A$ reactions [3]. The problem has been studied in effective hadronic models and QCD sum rules (QSR) generalized to finite baryon density. In QSR, it was shown that the vector meson mass drops to about 10-20 $\%$ of its vacuum value in nuclear matter density [4]. This is consistent with the idea of the Brown-Rho scaling [5] and also with Walecka model calculations including the vacuum polarization [6].

Recently, Koike [7] claimed that the previous QSR calculation for vector meson in nuclear medium [4] is not correct and that the vector meson mass should increase slightly. Since both ref. [4] and [7] on the vector meson masses are based on the Fermi gas approximation for nuclear matter, it is desirable to clarify the origin of the difference. In this work, we will first give a correct account of the Fermi-gas approximation of the QSR in medium and show dangers to relate mass shifts with the scattering length. Secondly, we will show that the approach in [7] is technically wrong so that in a correct application of QSR in medium [4] the vector meson mass does decrease in nuclear medium.

This paper is organized as follows. In section II, we will summarize the results of ref. «] and make new remarks which are not given in [4] but are relevant for the later discussions. In section III, the essential points of the Koike's claim are summarized. Section VI is devoted to critical investigation of [7]. We will show that his method is mortally flawed. In section $\mathrm{V}$, further elaboration of ref. [4] is discussed.

\section{QSR IN NUCLEAR MEDIUM}

Let's start with the retarded current correlation function in nuclear matter,

$$
\Pi_{\mu \nu}^{R}\left(\omega, \mathbf{q} ; n_{B}\right)=i \int d^{4} x e^{i q x}\left\langle\mathrm{R} J_{\mu}(x) J_{\nu}(0)\right\rangle_{n},
$$

where $q^{\mu} \equiv(\omega, \mathbf{q})$ and $\mathrm{R} J_{\mu}(x) J_{\nu}(0) \equiv \theta\left(x^{0}\right)\left[J_{\mu}(x), J_{\nu}(0)\right]$ with the source currents $J_{\mu}$ defined as $J_{\mu}=\frac{1}{2}\left(\bar{u} \gamma_{\mu} u \mp \bar{d} \gamma_{\mu} d\right)\left(-(+)\right.$ is for the $\rho^{0}(\omega)$-meson). $n_{B}$ denotes the baryon number density and $\langle\cdot\rangle_{n}$ is the expectation value in the ground state of nuclear matter.

Although there are two independent invariants in medium (transverse and longitudinal polarization), they coincide in the limit $\mathbf{q} \rightarrow 0$ and reduce to $\Pi_{\mu \mu}^{R} /\left(-3 \omega^{2}\right) \equiv \Pi^{R}$. $\Pi^{R}$ satisfies the following dispersion relation,

$$
\operatorname{Re} \Pi^{R}\left(\omega^{2}\right)=\frac{1}{\pi} \mathrm{P} \int_{0}^{\infty} d u^{2} \frac{\operatorname{Im} \Pi^{R}(u)}{u^{2}-\omega^{2}}+\text { (subtraction). }
$$

In QSR, the spectral density $\operatorname{Im} \Pi^{R}$ is modeled with several phenomenological parameters, while $\operatorname{Re} \Pi^{R}$ is calculated using the operator product expansion (OPE). The phenomenological parameters are then extracted by matching the left and right hand side of (2) in the asymptotic region $\omega^{2} \rightarrow-\infty$. 
Although the nuclear matter ground state has complicated structure, the Fermi-gas approximation taking into account the Pauli principle among the nucleons is a good starting point. In this approximation, $\Pi_{\mu \nu}^{R}$ reads

$$
\Pi_{\mu \nu}^{R}\left(\omega, \mathbf{q} ; n_{B}\right)=\Pi_{\mu \nu}^{R}(\omega, \mathbf{q} ; 0)+\gamma \int^{p_{f}} \frac{d^{3} p}{(2 \pi)^{3} 2 E_{N}} T_{\mu \nu}^{R}(\omega, \mathbf{q} \mid \mathbf{p}),
$$

with

$$
T_{\mu \nu}^{R}(\omega, \mathbf{q} \mid \mathbf{p})=i \int d^{4} x e^{i q x}\left\langle N(\mathbf{p})\left|\mathrm{R} J_{\mu}(x) J_{\nu}(0)\right| N(\mathbf{p})\right\rangle .
$$

Here $\gamma$ is a degeneracy factor ( $\gamma=4$ in nuclear matter), $|N(\mathbf{p})\rangle$ is the nucleon state with four momentum $p^{\mu}=\left(E_{N}, \mathbf{p}\right)$ in the covariant normalization $\left\langle N(\mathbf{p}) \mid N\left(\mathbf{p}^{\prime}\right)\right\rangle=(2 \pi)^{3} 2 E_{N} \delta^{3}(\mathbf{p}-$ $\left.\mathbf{p}^{\prime}\right)$. The spin average for the nucleon state is assumed in (4). $T_{\mu \nu}^{R}(\omega, \mathbf{q} \mid \mathbf{p})$ is nothing but the spin-averaged forward scattering amplitude of the external current $J_{\mu}$ and the nucleon. In nuclear matter, $\mathbf{p}$ is integrated out in the range $0<|\mathbf{p}|<p_{f}$ ( $p_{f}$ is the fermi momentum).

The OPE for $\operatorname{Re} \Pi^{R}\left(\omega^{2}\right)$, which is the same with the OPE for the causal (Feynman) correlation $\Pi^{F}\left(\omega^{2}\right)$, has a general form at $\omega^{2} \equiv-Q^{2} \rightarrow-\infty$,

$$
\operatorname{Re} \Pi^{R}\left(\omega^{2} \rightarrow-\infty\right)=\sum_{i} \frac{1}{Q^{2 i}} a_{i}\left(Q^{2} ; \mu^{2}\right)\left\langle\mathcal{O}_{i}\left(\mu^{2}\right)\right\rangle_{n},
$$

where $\mu$ is the renormalization point of the local operators, which separates the hard scale $|\omega|$ and soft scales such as $\Lambda_{Q C D}$ and $p_{f}$. The Wilson coefficients do not depend on the medium effect and only the expectation values $\left\langle\mathcal{O}_{i}\left(\mu^{2}\right)\right\rangle_{n}$ have the $p_{f}$ dependence.

The local operators $\mathcal{O}_{i}\left(\mu^{2}\right)$ in the vector meson sum rule are essentially the same with those in the lepton-nucleon deep inelastic scattering (DIS) and can be characterized by their canonical dimension $(d)$ and the twist $(\tau=$ dimension-spin). They are given in 10 up to dimension 6 operators and we will not recapitulate them here. Since we are taking $\mathbf{q} \rightarrow 0$, eq. (5) is an asymptotic series in $1 / \omega^{2}$ or equivalently an expansion with respect to $d$.

Now let us examine the density dependence of $\left\langle\mathcal{O}_{i}\left(\mu^{2}\right)\right\rangle_{n}$. In the Fermi-gas approximation, it reads

$$
\langle\mathcal{O}\rangle_{n}=\langle\mathcal{O}\rangle_{0}+\gamma \int^{p_{f}} \frac{d^{3} p}{(2 \pi)^{3} 2 E_{N}}\langle N(\mathbf{p})|\mathcal{O}| N(\mathbf{p})\rangle
$$

For the scalar matrix elements such as $\langle N|\bar{q} q| N\rangle$ and $\left\langle N\left|G^{2}\right| N\right\rangle$, one can utilize the information of the $\pi-N$ sigma term $\Sigma_{\pi N}$ and the QCD trace anomaly [11]. The results are

$$
\langle\bar{u} u\rangle_{n}=\langle\bar{u} u\rangle_{0}+\frac{\Sigma_{\pi N}}{2 \hat{m}} \cdot n_{B} \cdot B_{1}(x), \quad\left\langle\frac{\alpha_{s}}{\pi} G^{2}\right\rangle_{n}=\left\langle\frac{\alpha_{s}}{\pi} G^{2}\right\rangle_{0}-\frac{8}{9} m_{N}^{(0)} \cdot n_{B} \cdot B_{1}(x) .
$$

Here $\hat{m}(1 \mathrm{GeV})=(7 \pm 2) \mathrm{MeV}$ is the average value of the current quark masses of $u$ and $d$ quarks. The parameters we use are $\Sigma_{\pi N}=(45 \pm 7) \mathrm{MeV},\left\langle\frac{\alpha_{s}}{\pi} G^{2}\right\rangle_{0}=(350 \mathrm{MeV})^{4},\langle\bar{u} u\rangle_{0}=$ $(-230 \mathrm{MeV})^{3}$ 幽 and $m_{N}^{(0)}=770-830 \mathrm{MeV}$ with $y \equiv 2\langle N|\bar{s} s| N\rangle /(\langle N|\bar{u} u| N\rangle+\langle N|\bar{d} d| N\rangle)=$ $0.22-0.12$ [4], [1]. Non-leading $p_{f}$ corrections are contained in $B_{1}(x)$ defined as

$$
B_{1}(x)=\frac{3}{2 x^{3}}\left(x \sqrt{1+x^{2}}-\ln \left[x+\sqrt{1+x^{2}}\right]\right) \simeq 1-\frac{3}{10} x^{2}+\frac{9}{56} x^{4} \cdots,
$$


where $x=p_{f} / m_{N}$ and $n_{B}=\gamma p_{f}^{3} /\left(6 \pi^{2}\right)$. For nuclear matter density $n_{0}=0.17 / \mathrm{fm}^{3}(x=$ $0.27), x$ dependence of $B_{1}(x)$ can be safely neglected. Note also that $\langle\bar{d} d\rangle_{n}=\langle\bar{u} u\rangle_{n}$, since we are considering the symmetric nuclear matter $(\mathrm{N}=\mathrm{Z})$.

As for the four quark condensate in medium, there are no experimental data available yet. Thus we use a simple mean field approximation in nuclear matter [4];

$$
\left\langle\left(\bar{q} \gamma_{\mu} \gamma_{5} \lambda^{a} q\right)^{2}\right\rangle_{n} \simeq-\left\langle\left(\bar{q} \gamma_{\mu} \lambda^{a} q\right)^{2}\right\rangle_{n} \simeq \frac{16}{9}\left[\left\langle(\bar{q} q)^{2}\right\rangle_{0}+2\langle\bar{q} q\rangle_{0}\langle N|\bar{q} q| N\rangle \cdot n_{B} \cdot B_{1}(x)\right]
$$

As for $\left\langle(\bar{q} q)^{2}\right\rangle_{0}$ at $1 \mathrm{GeV}$ scale, we will use the canonical value $(-281 \mathrm{MeV})^{6}$ [15] with $\alpha_{s}(1 \mathrm{GeV}) \simeq 0.36$. This number, which is substantially larger than the current algebra value $\langle\bar{q} q\rangle_{0} \simeq(-230 \mathrm{MeV})^{3}$ at $1 \mathrm{GeV}$, should be considered as an effective one containing non-leading $1 / N_{c}$ contributions. As for $\langle\bar{q} q\rangle_{0}$ in the second term of (9), it is not clear whether one should use $(-230 \mathrm{MeV})^{3}$ or $(-281 \mathrm{MeV})^{3}$. (Note that the latter number was used in [4.) Taking into account such ambiguity as well as the "experimental" errors of $\Sigma_{\pi N}$ and $\hat{m}$, we adopt $\langle\bar{q} q\rangle_{0}\langle N|\bar{q} q| N\rangle=(-256 \mathrm{MeV})^{3} \cdot(45 / 14) \cdot(1 \pm 0.368)$ as a standard value to be used in (9).

The twist-2 quark bilinear operators with dimension 4 and 6 have non- vanishing matrix elements in the medium. Their nucleon matrix elements are related to the parton distribution function in DIS as,

$$
\begin{array}{r}
\left.\left\langle N(p)\left|\mathcal{S} \mathcal{T} \bar{q} \gamma_{\mu_{1}} D_{\mu_{2}} \cdots D_{\mu_{n}} q\right| N(p)\right\rangle\right|_{\mu^{2}}=(-i)^{n-1} A_{n-1}^{q}\left(\mu^{2}\right) T_{\mu_{1} \cdots \mu_{n}}, \\
A_{n-1}^{q}\left(\mu^{2}\right)=2 \int_{0}^{1} d x x^{n-1}\left[q\left(x, \mu^{2}\right)+\bar{q}\left(x, \mu^{2}\right)\right] .
\end{array}
$$

Here $T_{\mu_{1} \cdots \mu_{n}} \equiv\left[p_{\mu_{1}} \cdots p_{\mu_{n}}-\right.$ (trace terms) $] / 2 m_{N}$ and $\mathcal{S T}$ makes the operators symmetric and traceless. For the parton distribution function $q\left(x, \mu^{2}\right)$, we take the LO scheme in ref. [12] at the scale $\mu^{2}=1 \mathrm{GeV}$ which is close to the relevant Borel mass. Then we obtain $A_{1}^{u+d} \simeq 0.9$, $A_{3}^{u+d} \simeq 0.12$ at $1 \mathrm{GeV}$. The fermi-motion corrections are contained in $B_{2}(x)=\sqrt{1+x^{2}}$ and $B_{3}(x)=\sqrt{1+x^{2}}\left(1+\frac{8}{5} x^{2}\right)$ for spin 2 and spin 4 operators respectively. The deviation of $B_{2,3}(x)$ from 1 is again small at nuclear matter density.

In reference 四, all the operators up to $d=6$ except for relatively small twist- 4 spin- 2 operators are taken into account. In section IV, we will discuss the effect of the twist-4 operators to the result of [4].

In the vacuum QSR, the spectral function (i.e. $\operatorname{Im} \Pi^{R}$ in eq.(2)) is modeled with a resonance pole and the continuum. In the medium, we have to add additional singularities below the lowest resonance pole within the Fermi-gas approximation, which is called the Landau damping contribution [13]. For $\mathbf{q} \rightarrow 0$, it is calculable exactly and behaves like a pole at $\omega^{2}=0$ (see Appendix A for the proof). In total, the hadronic spectral function looks as

$$
\begin{aligned}
8 \pi \operatorname{Im} \Pi^{R}\left(u>0^{-}\right) & =\delta\left(u^{2}\right) \rho_{s c}+F \delta\left(u^{2}-m_{V}^{2}\right)+\left(1+\frac{\alpha_{s}}{\pi}\right) \theta\left(u^{2}-S_{0}\right) \\
& \equiv \rho_{\text {had. }}\left(u^{2}\right),
\end{aligned}
$$

with $\rho_{s c}=2 \pi^{2} n_{B} / \sqrt{p_{f}^{2}+m_{N}^{2}} \simeq 2 \pi^{2} n_{B} / m_{N} \cdot m_{V}, F$ and $S_{0}$ are the three phenomenological parameters to be determined by the sum rules. 
Matching the OPE side and the phenomenological side via the dispersion relation in the asymptotic region $\omega^{2} \rightarrow-\infty$, we can relate the resonance parameters to the density dependent condensates. There are two major procedure for this matching, namely the finite energy sum rules (FESR) [14 and the Borel sum rules (BSR) 15 which are summarized as the following forms:

$$
\begin{aligned}
& \int_{0}^{\infty} d s W(s)\left[\rho_{\text {had. }}(s)-\rho_{O P E}(s)\right]=0 \\
& W(s)= \begin{cases}s^{n} \theta\left(S_{0}-s\right) & (\mathrm{FESR}), \\
e^{-s / M^{2}} & (\mathrm{BSR}) .\end{cases}
\end{aligned}
$$

Here the spectral function $\rho_{\text {had. }}(s)$ stands for eq.(11). $\rho_{O P E}(s)$ is a hypothetical imaginary part of $\Pi^{R}$ which, through the dispersion relation (2), reproduces eq.(5). For more details on (12) and the explicit form of $\rho_{O P E}$, see section 2 of ref. [10.

\section{FESR and BSR for $\Pi^{R}\left(\omega^{2}\right)$}

First, for the qualitative argument, let us write down the FESR for the rho (omega) meson in the chiral limit. This can be easily obtained by taking the first three moments $n=0,1,2$ in (12):

$$
\begin{gathered}
F-S_{0}\left(1+\frac{\alpha_{s}}{\pi}\right)=-2 \pi^{2} m_{N}^{-1} \cdot n_{B} \quad(n=0), \\
F m_{V}^{2}-\frac{S_{0}^{2}}{2}\left(1+\frac{\alpha_{s}}{\pi}\right)=-\mathcal{Q}_{4}-2 \pi^{2} A_{1}^{u+d} m_{N} n_{B} \equiv-\tilde{\mathcal{Q}}_{4} \quad(n=1), \\
F m_{V}^{4}-\frac{S_{0}^{3}}{3}\left(1+\frac{\alpha_{s}}{\pi}\right)=-\mathcal{Q}_{6}-\frac{10}{3} \pi^{2} A_{3}^{u+d} m_{N}^{3} n_{B} \equiv-\tilde{\mathcal{Q}}_{6} \quad(n=2),
\end{gathered}
$$

where $\mathcal{Q}_{4}\left(\mathcal{Q}_{6}\right)$ is $\frac{\pi^{2}}{3}\left\langle\frac{\alpha_{s}}{\pi} G^{2}\right\rangle_{n}\left(\frac{896}{81} \pi^{3}\left\langle\alpha_{s}(\bar{q} q)^{2}\right\rangle_{n}\right)$ taken up to linear in $n_{B}$. Using the three relations above, we can determine the three phenomenological parameters $F, S_{0}$ and $m_{V}$ or equivalently, the changes from the vacuum values $\delta F, \delta S_{0}$ and $\delta m_{V}$. Important density dependence comes from $A_{1}^{u+d} n_{B}$ and the the 4-quark condensate $\mathcal{Q}_{6}$, which can be shown by solving eq.(13) numerically.

Although both FESR and BSR give the same qualitative result, BSR is more reliable for the quantitative estimate since it is rather insensitive to the assumption on the continuum. The rho (omega) meson mass in the BSR is given as

$$
\frac{m_{V}^{2}}{M^{2}}=\frac{\left(1+\frac{\alpha_{s}}{\pi}\right)\left(1-e^{-S_{0} / M^{2}}\left(1+\frac{S_{0}}{M^{2}}\right)\right)-\frac{1}{M^{4}} \tilde{\mathcal{Q}}_{4}+\frac{1}{M^{6}} \tilde{\mathcal{Q}}_{6}}{\left(1+\frac{\alpha_{s}}{\pi}\right)\left(1-e^{-S_{0} / M^{2}}\right)+\frac{1}{M^{4}} \tilde{\mathcal{Q}}_{4}-\frac{1}{2 M^{6}} \tilde{\mathcal{Q}}_{6}-\rho_{s c}} .
$$

In Fig.1, the Borel curve $\left(m_{V}-M^{2}\right.$ relation) is shown for different baryon densities. The continuum threshold $S_{0}$ is chosen to make the Borel curve as flat as possible in the Borel window $M_{\min }^{2}<M^{2}<M_{\max }^{2}$ at given density. We take density-independent window $M_{\min }^{2}=$ $0.41 \mathrm{GeV}^{2}$ and $M_{\max }^{2}=1.30 \mathrm{GeV}^{2}$ in our analyses. More general procedure with densitydependent window (see e.g. section 4 of ref. [10]) does not change the results quantitatively. 


\section{Fig.1}

By making a linear fit using the values at $n=n_{0}$ and $n=n_{B}$, we get

$$
\begin{aligned}
\frac{m_{V}\left(n_{B}\right)}{m_{V}(0)} & =1-(0.16 \pm 0.06) \frac{n_{B}}{n_{0}}, \\
\sqrt{\frac{S_{0}\left(n_{B}\right)}{S_{0}(0)}} & =1-(0.15 \pm 0.05) \frac{n_{B}}{n_{0}}, \\
\frac{F\left(n_{B}\right)}{F(0)} & =1-(0.24 \pm 0.07) \frac{n_{B}}{n_{0}},
\end{aligned}
$$

These values are slightly different from our previous ones in [4] where the uncertainty discussed below (9) is not taken into account. For the decreasing rho (omega) mass, the twist 2 and the scalar matrix elements are equally important.

\section{MASS SHIFT AND THE SCATTERING LENGTH}

In this section, we will first summarize the claims given explicitly or implicitly in [7]. Although they look plausible in the first look, every statement is invalid as we will show in section III.A-C.

(A) Koike starts with eq.(33) and makes a low density approximation for the second term

$$
\gamma \int^{p_{f}} \frac{d^{3} p}{(2 \pi)^{3} 2 E_{N}} T_{\mu \nu}^{R}(\omega, \mathbf{q} \mid \mathbf{p}) \rightarrow n_{B} \frac{T_{\mu \nu}^{R}(\omega, \mathbf{q} \mid \mathbf{0})}{2 m_{N}} .
$$

This corresponds to the assumption that all the nucleons in nuclear medium are at rest $(\mathbf{p}=0)$. If one further takes the kinematics $\mathbf{q}=0$ and $\omega \simeq m_{V}, T^{R} \equiv T_{\mu \mu}^{R}(\omega, \mathbf{q}=0 \mid \mathbf{0})$ is written by the $V-N$ scattering length as

$$
T^{R} \simeq \frac{3 F m_{V}^{2}}{8 \pi^{2}} \frac{24 \pi\left(m_{N}+m_{V}\right) a_{V N}}{\left(\omega^{2}-m_{V}^{2}\right)^{2}}+\mathrm{R}\left(\omega^{2}\right),
$$

where $a_{V N}=\left(a_{1 / 2}+2 a_{3 / 2}\right) / 3$ with $a_{1 / 2}$ and $a_{3 / 2}$ being the $V-N$ scattering length in the spin- $1 / 2$ and spin- $3 / 2$ channel respectively. $\mathcal{R}\left(\omega^{2}\right)$ is the term less singular than the leading double-pole term in the Laurent expansion around $\omega^{2}=m_{V}^{2}$.

By substituting these expressions to eq.(3) and take the leading term in eq.(19), one arrives at the formula

$$
\begin{aligned}
\frac{1}{3} \Pi_{\mu \mu}^{R}\left(\omega \simeq m_{V}, \mathbf{q}=0 ; n_{B}\right) & \simeq \frac{F m_{V}^{2}}{8 \pi^{2}}\left(\frac{1}{\omega^{2}-m_{V}^{2}}+\frac{12 \pi a_{V N}\left(m_{N}+m_{V}\right) / m_{N}}{\left(\omega^{2}-m_{V}^{2}\right)^{2}} n_{B}\right), \\
& \propto \frac{1}{\omega^{2}-\left(m_{V}+\delta m_{V}\right)^{2}}
\end{aligned}
$$

with 


$$
\delta m_{V}=6 \pi \frac{m_{N}+m_{V}}{m_{N} m_{V}} \cdot a_{V N} \cdot n_{B} .
$$

Hence the positive (negative) scattering length gives an increasing (decreasing) mass in the medium.

(B) To estimate the magnitude and sign of the scattering length $a_{V N}$ in (21), Koike formulated QCD sum rules for $T^{R}$. He starts with the unsubtracted dispersion relation;

$$
\operatorname{Re} T^{R}\left(\omega^{2}\right)=\frac{1}{\pi} \int_{0}^{\infty} d u^{2} \frac{\operatorname{Im} T^{R}(u)}{u^{2}-\omega^{2}}
$$

The OPE for $T^{R}$ is expanded up to $O\left(1 / Q^{4}\right)$ as

$$
\operatorname{Re} T_{O P E}^{R}\left(Q^{2}\right)=\frac{1}{8 \pi^{2}}\left(\frac{c_{1}}{Q^{2}}-\frac{c_{2}}{Q^{4}}\right)
$$

where $c_{1,2} / 2 m_{N} \equiv d \tilde{\mathcal{Q}}_{4,6} / d n_{B}$ at $n_{B}=0$. The absence of the logarithmic term in (23) indicates that subtraction is not necessary in (22). Motivated by eq.(19), the imaginary part is parameterized as

$$
8 \pi \operatorname{Im} T^{R}(u>0)=b_{1} \delta^{\prime}\left(u^{2}-m_{V}(0)^{2}\right)+b_{2} \delta\left(u^{2}-m_{V}(0)^{2}\right)+b_{3} \delta\left(u^{2}-S_{0}(0)\right),
$$

with three unknowns $b_{1,2,3}$ and known vacuum parameters $m_{V}(0)$ and $S_{0}(0)$. (Note that $\left.b_{1}=F m_{V}^{2} 24 \pi\left(m_{N}+m_{V}\right) a_{V N}.\right)$ Above parametrization is equivalent to taking the following ansatz for the real part

$$
\operatorname{Re} T_{h a d .}^{R}\left(Q^{2}\right)=\frac{b_{1}}{\left(m_{V}(0)^{2}+Q^{2}\right)^{2}}+\frac{b_{2}}{m_{V}(0)^{2}+Q^{2}}+\frac{b_{3}}{S_{0}(0)+Q^{2}} .
$$

By constructing a borel sum rule using (22) - (24), Koike obtains positive scattering length $a_{\rho(\omega)} \simeq 0.14(0.11) \mathrm{fm}$, from which he concluded that the mass shift $\delta m_{V}$ in eq.(21) must be positive.

(C) Koike further claims that the above procedure $(A)+(B)$ is equivalent with doing the medium sum rule for $\Pi_{\mu \mu}^{R}$ but not for $\Pi^{R}$. (Note that $\Pi_{\mu \mu}^{R}=-3 \omega^{2} \Pi^{R}$ when $\left.\mathbf{q}=0\right)$. This can be seen as follows. The dispersion relation in medium for $\Pi_{\mu \mu}^{R}$ reads

$$
\operatorname{Re} \Pi_{\mu \mu}^{R}\left(\omega^{2}\right)=\frac{1}{\pi} \mathrm{P} \int_{0}^{\infty} d u^{2} \frac{\operatorname{Im}\left[\Pi_{\mu \mu}^{R}(u)\right]}{u^{2}-\omega^{2}}+(\text { subtraction })
$$

If one adopts eq. (11) for $\operatorname{Im} \Pi^{R}$ and uses the relation $\Pi_{\mu \mu}^{R}=-3 \omega^{2} \Pi^{R}$, one obtains

$$
\begin{aligned}
\left(-\frac{1}{3}\right) 8 \pi \operatorname{Im}\left[\Pi_{\mu \mu}^{R}(u)\right] & =F m_{V}^{2} \delta\left(u^{2}-m_{V}^{2}\right)+\left(1+\frac{\alpha_{s}}{\pi}\right) u^{2} \theta\left(u^{2}-S_{0}\right), \\
& =u^{2} \rho_{\text {had. }}\left(u^{2}\right) .
\end{aligned}
$$

Since $u^{2} \delta\left(u^{2}\right)=0$, the Landau damping term in (11) does not arise in (27). 
Expansion of the 1.h.s. of (26) in terms of $n_{B}$ gives $\left[n_{B}\right.$ independent term $]+\left[n_{B} \times\right.$ eq.(23)], while the same expansion of (27) gives $\left[n_{B}\right.$ independent term $]+\left[n_{B} \times\right.$ eq.(24)]. The latter is obtained simply by writing $m_{V}=m_{V}(0)+\delta m_{V}, S_{0}=S_{0}(0)+\delta S_{0}, F=F(0)+\delta F$, expanding (27) up to linear in $\delta m_{V}, \delta F$ and $\delta S_{0}$ and doing the following identification.

$$
b_{1} / 2 m_{N}=-F m_{V}^{2} \delta m_{V}^{2}, \quad b_{2} / 2 m_{N}=m_{V}^{2} \delta F+F \delta m_{V}^{2}, \quad b_{3} / 2 m_{N}=-S_{0} \delta S_{0} .
$$

This means that the sum rule for $T^{R}$ eq.(22) is equivalent to the linear density part of the sum rule for $\Pi_{\mu \mu}^{R}$ eq.(26). Assuming that his procedure $(\mathrm{A})+(\mathrm{B})$ is right, Koike thus concluded that (i) the medium QSR using $\Pi_{\mu \mu}^{R}$ must give the increasing vector-meson mass, and (ii) the result of the medium sum rule using $\Pi^{R}$ in [4] must be wrong.

In the following, we will discuss that each of the above arguments is invalid. The subsection numbers III.A, III.B and III.C in the following correspond to the statements (A), (B) and (C) in the above, respectively.

\section{A. Mass shift and the scattering length}

First of all, let us show that eq.(18) is a useless approximation around nuclear matter density. In eq.(18), the motion of nucleons and the Pauli exclusion principle in nuclear matter are completely neglected. Such approximation is valid only when (i) the nucleon density is extremely low, or (ii) $T_{\mu \nu}^{R}(\omega, \mathbf{q}=0 \mid \mathbf{p})$ is almost constant as a function of $\mathbf{p}$ in the interval $0<|\mathbf{p}|<p_{f}$.

Since we are not interested in the case (i), let us concentrate on (ii) and see whether (ii) is plausible or not. At nuclear matter density, the fermi momentum is sizable $p_{f} \simeq 270 \mathrm{MeV}$. Thus we should consider e.g. the $\rho-N$ scattering from $\sqrt{s}=m_{\rho}+m_{N}=1709 \mathrm{MeV}$ through $\sqrt{s}=\left(\left(m_{\rho}+\sqrt{m_{N}^{2}+p_{f}^{2}}\right)^{2}-p_{f}^{2}\right)^{1 / 2}=1726 \mathrm{MeV}$. In this interval, there are at least two schannel resonances $N(1710)$ and $N(1720)$ and also there are two nearby resonances just below the threshold $N(1700)$ and $\Delta(1700)$ [18]. They are all possible to have coupling with $\rho N$. This means that $T_{\mu \nu}^{R}$ has a rapid variation as a function of $|\mathbf{p}|$ between $|\mathbf{p}|=0$ and $|\mathbf{p}|=p_{f}$ due to the effect of these s-channel resonances and it is impossible to approximate it by the threshold value (i.e. the $V-N$ scattering length) $T^{R}\left(\omega=m_{V}, \mathbf{q}=0 \mid \mathbf{p}=0\right)$. See Fig.2 for a schematic illustration of the $s$-channel contributions [16].

\section{Fig.2}

What one can expect at best is the approximate linear density formula written in terms of the average of $T_{\mu \nu}^{R}$ in the region $0<|\mathbf{p}|<p_{f}$ :

$$
\gamma \int^{p_{f}} \frac{d^{3} p}{(2 \pi)^{3} 2 E_{N}} T_{\mu \nu}^{R}(\omega, \mathbf{q}=0 \mid \mathbf{p}) \simeq n_{B}\left\langle\frac{T_{\mu \nu}^{R}(\omega, \mathbf{q}=0 \mid \mathbf{p})}{2 E_{N}}\right\rangle
$$

where $\langle\cdot\rangle$ stands for the average over the above momentum interval.

It is easy to see what is wrong in (18) in physical terms: the rho-meson at rest $(\mathbf{q}=0)$ in nuclear matter will suffer the scattering from the nucleons having various three-momentum $\mathbf{p}$ in the interval $0<|\mathbf{p}|<p_{f}$. The net effect should be the averaged strength of the scattering and not the scattering length defined at $\mathbf{p}=0$. This is particularly so when $T_{\mu \nu}^{R}$ has a rapid $\mathbf{p}$ dependence. 
The above point is well known for the nucleon in nuclear medium. The optical potential for the nucleon at rest in nuclear matter cannot be approximated by the $\mathrm{N}-\mathrm{N}$ scattering length multiplied by the nuclear density. In fact, the $\mathrm{N}-\mathrm{N}$ forward scattering amplitude $T_{N N}(p)$ has a huge momentum dependence due to the the deuteron state and the "almost" bound state near the threshold. The relevant quantity for the nucleon optical potential is not the scattering length but the averaged scattering amplitude in the interval $0<|\mathbf{p}|<p_{f}$. This point was recently emphasized by Furnstahl and one of the present authors [9]: They criticise the paper by Kondo and Morimatsu [17] who use a similar approximation with (18) to analyse the nucleon in nuclear medium.

It is now clear that the mass shift and the scattering length cannot be related directly at nuclear matter density. In the approach of ref. [4], neither eq.(18) nor eq.(29) are adopted, thus one does not suffer from this problem.

\section{B. Is scattering length calculable in QSR?}

Since eq.(18) and hence eq.(21) are not valid around nuclear matter density, it is useless to relate the scattering length with the mass shift. Nevertheless, the $V-N$ scattering length itself could be an interesting physical quantity to be calculated in QSR. We will show, however, that the method in ref. [7] to estimate $a_{V N}$ is erroneous.

Let us look at (23) and (25) and compare them at $Q^{2} \rightarrow \infty$, which corresponds to the FESR for $T^{R}$. One immediately realizes that only two independent equations can be obtained

$$
\begin{array}{r}
b_{2}+b_{3}=c_{1}, \\
b_{1}-m_{V}(0)^{2} b_{2}-S_{0}(0) b_{3}=-c_{2},
\end{array}
$$

whereas one needs three equations to solve $b_{1,2,3}$. This happens because the OPE is calculated only up to $O\left(1 / Q^{4}\right)$ in (23). (30) clearly shows that it is impossible to predict the scattering length $a_{V N}$ (which is proportional to $b_{1}$ ).

In QCD sum rules, one should always check that the number of phenomenological parameters to be determined is equal or smaller than the number of OPE terms, otherwise sum rules are not closed and cannot give predictions. The FESR provides a useful tool to do this consistency check.

If one tries to make Borel analyses without the consistency as has been done in ref. [7], one simply obtains a fake result and does not get any stability of the Borel curve. By blindly doing a Borel sum rule without looking for a stability region, Koike obtains a positive number for $b_{1}$, in which it is implicitly assumed that the contribution from dimension 8 operators is zero. In terms of finite energy sum rule, this amounts to the following condition,

$$
2 m_{V}(0)^{2} b_{1}-m_{V}(0)^{4} b_{2}+S_{0}(0)^{2} b_{3}=0 .
$$

With this assumption and eq.(30), we have three equations and the unknown constants can be determined. Using the fact that $c_{1} \sim 0, c_{2}>0$ and $S_{0}>m_{V}^{2}$, one finds a positive value for $b_{1}$. However, the assumption eq.(31) has no ground and we do expect non-negligible contribution from the dimension 8 operators. Since it is technically very hard to calculate these dim. 8 condensates $\left(1 / Q^{6}\right.$ terms of $\left.T^{R}\right)$, it is almost hopeless to get reliable $a_{V N}$ in QSR. 


\section{Use of QSR for $\Pi_{\mu \mu}^{R}$}

Here, we will explicitly demonstrate that the sum rules for $\Pi_{\mu \mu}^{R}$ recommended in [7] does not work at all. Let's first start with the FESR for $\Pi_{\mu \mu}^{R}$;

$$
\int_{0}^{S_{0}} d s s^{n}\left[\operatorname{Im} \Pi_{\mu \mu}^{R}(s)_{h a d .}-\operatorname{Im}_{\mu \mu}^{R}(s)_{O P E}\right]=0 \quad(n=0,1,2, \cdots) .
$$

Then one immediately finds that only two relations corresponding to $n=0,1$ are obtained, and they turn out to be equivalent with the second and third relations in eq.(13). (Note that $\mathrm{n}=2$ in (32) cannot gives another condition because OPE is calculated only up to dimension 6 operators.)

$$
\begin{aligned}
& F m_{V}^{2}-\frac{S_{0}^{2}}{2}\left(1+\frac{\alpha_{s}}{\pi}\right)=-\tilde{\mathcal{Q}}_{4}, \quad(n=0) \\
& F m_{V}^{4}-\frac{S_{0}^{3}}{3}\left(1+\frac{\alpha_{s}}{\pi}\right)=-\tilde{\mathcal{Q}}_{6} \quad(n=1) .
\end{aligned}
$$

There are three unknowns $F, m_{V}, S_{0}$, while only two relations are available. Thus unless one introduces extra assumption, it is impossible to solve for three even in the vacuum. This is exactly the same problem which we have discussed in sec.III.B.

One should also note that the missing condition is the duality relation for the spectral density:

$$
\int_{0}^{S_{0}} d s\left[\rho_{\text {had. }}(s)-\rho_{O P E}(s)\right]=0
$$

with $\rho_{\text {had. }}$ and $\rho_{O P E}$ being the spectral densities for $\Pi^{R}$. This local duality is the cornerstone of vacuum QSR and holds also in medium since there are no dimension 2 operators in OPE. If one wants to get reliable result from FESR, One has either to work out the OPE up to dimension 8 operators (which is a formidable task) or to start with $\Pi^{R}$ as in ref. [1].

Here one may ask that "why not take $n=-1$ moment in (32) to obtain another relation?". Such procedure, however, introduces an ambiguity at $s=0$, since one can add any function proportional to $s \delta(s)$ to $\operatorname{Im} \Pi_{\mu \mu}^{R}$ which does not modify $n=0,1$ sum rules but modifies $n=-1$ sum rule. If one tries to remove this ambiguity, it is necessary to start with $\Pi^{R}$ and to evaluate the Landau damping term in (11) which is exactly calculable as we mentioned [19].

Let us now turn to the BSR for $\Pi_{\mu \mu}^{R}$ :

$$
\frac{m_{V}^{2}}{M^{2}}=\frac{2\left(1+\frac{\alpha_{s}}{\pi}\right)\left(1-e^{-S_{0} / M^{2}}\left(1+\frac{S_{0}}{M^{2}}+\frac{S_{0}}{2 M^{2}}\right)\right)-\frac{1}{M^{6}} \tilde{\mathcal{Q}}_{6}}{\left(1+\frac{\alpha_{s}}{\pi}\right)\left(1-e^{-S_{0} / M^{2}}\left(1+\frac{S_{0}}{M^{2}}\right)\right)-\frac{1}{M^{4}} \tilde{\mathcal{Q}}_{4}+\frac{1}{M^{6}} \tilde{\mathcal{Q}}_{6}}
$$

Eq.(14) and eq.(35) in this paper correspond to eq.(13) and eq.(14) in [7] respectively. Since $\tilde{\mathcal{Q}}_{6}$ enters with opposite sign in the r.h.s. of eq.(14) and eq.(35) and $\tilde{\mathcal{Q}}_{6}$ decreases in medium, Koike simply concluded that $m_{V}$ decreases in (14), while it increases in (35). This conclusion is too naive: in fact, $S_{0}$ in the r.h.s. of these equations is also density dependent, which can 
change such naive expectation. density dependence of $S_{0}$ can be in principle determined by the Borel stability procedure we have discussed in section II.

In order to see whether this procedure works or not for eq.(35), we have shown the Borel curves for different values of $S_{0}$ in Fig. 3(a) (at zero density) and in Fig.3 (b) (at nuclear matter density). Fig. 3(a) shows that the Borel curve does not have any plateau in the relevant range of $M^{2}$ (say $0.41<M^{2}<1.30$ ), which implies that one cannot determine $S_{0}$ and hence $m_{V}$ even in the vacuum. The situation is the same at the nuclear matter density as is shown in Fig.3(b). Again, one cannot determine $S_{0}\left(n_{0}\right)$ by the Borel stability method and hence $m_{V}\left(n_{0}\right)$, which implies that there is no hope to determine the mass shift at finite density. If one sticks to a specific value of $S_{0}$ (say $2.0 \mathrm{GeV}^{2}$ ) and uses it at any density, one finds that $m_{V}\left(n_{B}\right) / m_{V}(0)>1$ for given $M^{2}$ from Fig.3. This is equivalent with the "naive" (and wrong) argument by Koike. The correct procedure is to compare $m_{V}\left(n_{B}\right)$ (calculated with $S_{0}\left(n_{B}\right)$ ) and $m_{V}(0)$ (calculated with $S_{0}(0)$ ).

\section{Fig.3}

The "bad" Borel curves in Fig. 3 is quite in contrast to the "good" Borel curves for $\Pi^{R}$ in Fig.1. The latter shows beautiful stability in the vacuum as well as in the medium, which makes one possible to determine $S_{0}\left(n_{B}\right)$ at each density and hence $m_{V}\left(n_{B}\right)$.

The reason of the failure of the BSR for $\Pi_{\mu \mu}^{R}$ is twofold. Firstly, the higher dimensional operators in OPE is rather important for $\Pi_{\mu \mu}^{R}$ sum rules. We have already seen this in (33) where $n=2$ sum rule can be obtained only when one has dim. 8 operators in OPE. In BSR, the lack of the information of dim. 8 operators arises as a instability of the Borel curve at low $M$ region. Inclusion of the dim. 8 operator would make the curve more flat. Secondly, the continuum contribution is more important in $\Pi_{\mu \mu}^{R}$ than in $\Pi^{R}$, since the spectral function is increasing linearly in the former case. This makes the prediction of the resonance parameters less reliable in the former.

Let us summarize here the lessons we learned in subsections III.A, III.B and III.C. Firstly, the mass shift and the scattering length does not have direct relation in nuclear matter due to the momentum dependence of the $V-N$ forward scattering amplitude. Secondly, sum rules for the $V-N$ scattering amplitude cannot predict the $V-N$ scattering length without dimension 8 operators in OPE. Thirdly, sum rules for $\Pi_{\mu \mu}^{R}$ does not work at all even in the vacuum without dimension 8 operators in OPE. Thus all the claims in ref. [7] are shown to be erroneous. Also, only the consistent QSR in medium currently available is the one starting from $\Pi^{R}$ given in ref. 四.

\section{SEVERAL COMMENTS}

\section{Full twist 4 calculation}

There are three kinds of twist- 4 spin- 2 operators contributing to the $\rho, \omega$ sum rules. At present, their nucleon matrix elements are not known. However, the values of two different combinations in the transverse and longitudinal structure functions of the nucleon have been obtained by two of us 20,21 by analyzing the recent DIS data at CERN and SLAC. Let us further make the following assumption [20]; 


$$
\frac{\left\langle\bar{d} \Gamma_{\mu} \Delta_{\nu} d\right\rangle}{\left\langle\bar{u} \Gamma_{\mu} \Delta_{\nu} u\right\rangle}=\frac{\left\langle\bar{d} \gamma_{\mu} D_{\nu} d\right\rangle}{\left\langle\bar{u} \gamma_{\mu} D_{\nu} u\right\rangle} \equiv \beta,
$$

where $\Gamma_{\mu}$ is some gamma matrix and $\Delta_{\mu}$ an isospin singlet operator. Then, it is possible to uniquely determine the nucleon matrix element of twist- 4 spin- 2 operators appearing in the $\rho, \omega$ sum rule from combinations of experimental values. It gives the following contribution to eq.(4),

$$
T_{\mu \mu}^{R, \tau=4}(\omega, \mathbf{q} \mid \mathbf{p})=\frac{-\left[(q \cdot p)^{2}-\frac{1}{4} m_{N}^{2} q^{2}\right]}{m_{N} Q^{4}}\left(-(1+\beta)\left(K_{u}^{1}+\frac{1}{4} K_{u}^{2}+\frac{5}{8} K_{u}^{g}\right)+K_{u d} \pm K_{u d}\right)
$$

The $K_{u}^{i} i=1,2, g$ are defined in ref. [20] and $-(+)$ corresponds to the $\omega(\rho)$ case. Choosing $\beta=0.476$ as in ref. [20] the value inside the large round bracket is $A^{4}=0.40$ (0.24) $\mathrm{GeV}^{2}$ for the $\omega(\rho)$ meson.

Now the effect of twist-4 matrix element can be estimated by making the following substitution for the dimension six operators.

$$
\left(\mathcal{Q}_{6}+\frac{10}{3} \pi^{2} A_{3}^{u+d} m_{N}^{3} n_{B}\right) \rightarrow\left(\mathcal{Q}_{6}+\frac{10}{3} \pi^{2} A_{3}^{u+d} m_{N}^{3} n_{B}+2 \pi^{2} m_{N} A^{4} n_{B}\right)
$$

The net effect of twist- 2 + twist-4 is estimated to be 2.36 (3.29) times larger than the twist 2 effect alone in the rho (omega) channel. This could change the slope of the mass shift in (15) from 0.16 to $0.10(0.075)$ for the rho (omega) meson. Further investigation is necessary however to draw definite conclusion on the magnitude of the twist 4 effect.

\section{Fermi momentum correction}

The small higher density effects coming from fermi-momentum correction can be estimated by looking at $B_{i}(x)$. One easily finds that $B_{1}(0.27)=0.979, B_{2}(0.27)=$ $1.036, B_{3}(0.27)=1.157$ and $B_{1}(0.34)=0.967, B_{2}(0.34)=1.056, B_{3}(0.34)=1.251$, where $x=0.27(0.34)$ corresponds to nuclear matter (twice of nuclear matter) density. Thus the effect can be safely neglected at nuclear matter density.

\section{Possible new structure in $\operatorname{Im} \Pi^{R}$}

It is possible that the density dependent change of the OPE side is balanced by some new structure appearing in the spectral density below the resonance mass. In QCD sum rule approach, it has to be included by hand before matching to the OPE. Such possibility has been examined by Asakawa and Ko [22] by redoing the medium QCD sum rules for the vector meson including other complex structure of the spectral density in the nuclear medium induced by the $\pi, \Delta, N, \rho$ dynamics. They found that even in that case the vector meson mass has to decrease in order to be consistent with the OPE side.

\section{$\phi$ meson sum rule}

The formalism for calculating the change of $\phi$ meson sum rule is the same as that of the $\rho$ and $\omega$. However, in the $\phi$ case, one must include the effect of the strange quark mass in the OPE and this will introduce some basic difference [1]. In the $\rho, \omega$ sum rule, the density dependence in OPE is dominated by $(d, \tau)=(4,2)$ and $(d, \tau)=(6,0)$ operators. However, in the case of the $\phi$ meson, the dimension 4 strange quark condensate $\left\langle m_{s} \bar{s} s\right\rangle$ is 
not suppressed either by $1 / 4 \pi^{2}$ or the light current quark mass and consequently dominates the OPE. In the medium, the change of this condensate, which comes from the the K-N sigma term, dominates the small changes in other condensate and introduce a non-negligible mass reduction by $3 \sim 5 \%$ [4]. It is amusing to compare this result with that of an effective model calculations [23] in which the $\mathrm{K}-\mathrm{N}$ sigma term also induces a small reduction of the $\phi$ mass.

\section{SUMMARY}

To estimate the mass shift of vector mesons in medium, we have carried out a detailed comparision between the approach based on the modification of the vacuum QSR [1] and that based on the scattering length [7]. We have shown that the latter approach is erroneous by the following reasons:

(i) The mass shift and the scattering length does not have direct relation in nuclear matter due to the momentum dependence of the $V-N$ forward scattering amplitude.

(ii) Sum rules for the $V-N$ scattering amplitude cannot predict the $V-N$ scattering length if dimension 8 operators in OPE are not included.

(iii) Sum rules for $\Pi_{\mu \mu}^{R}$ have no predictive power both in the vacuum and in the medium if dimension 8 operators are not included.

\section{ACKNOWLEDGMENTS}

The authors thank Dr. Y. Koike for stimulating discussions to clarify the issue, although he does not share our arguments. TH thanks Dr. K. Tanaka for useful comments and Prof. M. Ichimura for discussions and useful information on nucleons in nuclear matter. TH and SHL thank the Institute for Nuclear Theory at the University of Washington for its hospitality and the Department of Energy for partial support during the completion of this work. The work of TH and HS was supported in part by the Grants-in-Aids of the Japanese Ministry of Education (No. 06102004). The work of SHL was supported by the Basic Science Research Institute program of the Korean Ministry of Education through Grant No. BSRI-94-2425 and by KOSEF through the CTP at Seoul National University. 


\section{APPENDIX: A}

Here, we derive the Landau damping term $\rho_{s c}$. In the Fermi gas approximation, the spectral density has two types of contribution. The annihilation term, which is non-zero above the two particle threshold $\omega^{2}>\mathbf{q}^{2}+4 m_{N}^{2}$, and the scattering term, which is non-zero in the space-like region $\omega^{2}<\mathbf{q}^{2}$. We are interested in the second term. For finite $\mathbf{q}$, the spectral density, contributing to the longitudinal polarization, can be obtained by looking at the 00 component of the imaginary part of eq.(11).

$$
\rho_{l}^{s}(\omega, \mathbf{q})=\frac{\operatorname{Im} \Pi_{00}}{\mathbf{q}^{2}} \quad \stackrel{\mathbf{q} \rightarrow 0}{\longrightarrow} \quad \frac{\operatorname{Im} \Pi_{\mu \mu}}{-3 \omega^{2}} .
$$

Looking at the spectral representation, it is easy to identify the following scattering contribution,

$$
\begin{aligned}
\rho_{l}^{s}(\omega, \mathbf{q})=\frac{(2 \pi)^{4}}{4 \mathbf{q}^{2}} \int \frac{d^{3} k_{1}}{(2 \pi)^{3} E_{1}} & \frac{d^{3} k_{2}}{(2 \pi)^{3} E_{2}}\left|\left\langle N\left(\mathbf{k}_{1}\right)\left|J_{0}\right| N\left(\mathbf{k}_{2}\right)\right\rangle\right|^{2} \\
\times & \delta\left(\omega-E_{1}+E_{2}\right) \delta^{3}\left(\mathbf{q}-\mathbf{k}_{\mathbf{1}}+\mathbf{k}_{\mathbf{2}}\right)\left[n_{F}\left(E_{2}\right)-n_{F}\left(E_{1}\right)\right] .
\end{aligned}
$$

Here, $E_{i}=\sqrt{\mathbf{k}_{i}^{2}+m_{N}^{2}}(\mathrm{i}=1,2)$, and the $n_{F}\left(E_{i}\right)=\theta\left(\sqrt{k_{f}^{2}+m_{N}^{2}}-E_{i}\right)$, where $k_{f}$ is the fermi momentum.

In general, the nucleon expectation of the isospin current has two form factors.

$$
\left\langle N\left(\mathbf{k}_{\mathbf{1}}\right)\left|J_{\mu}^{a}\right| N\left(\mathbf{k}_{\mathbf{2}}\right)\right\rangle=\bar{u}\left(\mathbf{k}_{\mathbf{1}}\right) \frac{\tau^{a}}{2}\left[F_{1}(q) \gamma_{\mu}+F_{2}(q) i \sigma_{\mu \nu} q^{\nu}\right] \bar{u}\left(\mathbf{k}_{\mathbf{2}}\right) .
$$

Substituting this into eq. A2 , one obtains

$$
\begin{aligned}
& \rho_{l}^{s}(\omega, \mathbf{q})=\frac{\gamma}{256 \pi^{2}} \int_{v}^{\infty} d x[\left.\left(1-x^{2}\right) F_{1}^{2}(q)+x^{2}\left(\omega^{2}-\mathbf{q}^{2}\right) F_{2}^{2}(q)\right] \\
& \times\left(2 n_{F}\left(\frac{|\mathbf{q}| x+\omega}{2}\right)-2 n_{F}\left(\frac{|\mathbf{q}| x-\omega}{2}\right)\right) \theta\left(\mathbf{q}^{2}-\omega^{2}\right),
\end{aligned}
$$

where $v=\left[1-4 m^{2} /\left(\omega^{2}-\mathbf{q}^{2}\right)\right]^{1 / 2}$.

Now, we want to take the limit $|\mathbf{q}| \rightarrow 0$. In this limit, the constraint $0<\omega^{2}<\mathbf{q}^{2}$ also forces $\omega$ to approach zero. Consequently, the contribution proportional to $F_{2}$ vanishes, because it is multiplied by either $\mathbf{q}^{2}$ or $\omega^{2}$. As for the other term proportional to $F_{1}(q)$, the integral becomes increasingly large as $|\mathbf{q}| \rightarrow 0$ such that the integrated quantity of $\rho_{l}^{s}(\omega, \mathbf{q})$ within the phase space for $\omega$ remains finite.

By integrating over this region with $|\mathbf{q}|$ finite and then taking the limit, we find

$$
\lim _{|\mathbf{q}| \rightarrow 0} \int_{0}^{\mathbf{q}^{2}} d \omega^{2} \rho_{l}^{2}(\omega, \mathbf{q})=\frac{\gamma}{12} \int \frac{d^{3} p}{(2 \pi)^{3} 2 E} n_{F}(E) v(3-v) \equiv \rho_{s c} / 8 \pi
$$

so that $\rho_{l}^{s}(\omega, p)$ effectively becomes a delta function. Thus the final result is that the spectral density reduces to

$$
\lim _{|\mathbf{q}| \rightarrow 0} \rho_{l}^{s}(\omega, \mathbf{q})=\delta\left(\omega^{2}\right) \rho_{s c} / 8 \pi
$$

It should be noted here that we did not make any approximation from (A2) through (A5), thus the result is exact. Also note that there arises no ambiguity from the nucleon form factor because $F_{1}(q=0)=1$. 


\section{Figure Captions}

Fig.1: Borel curve for $m_{V}\left(M^{2}\right)$ using $\Pi^{R}$. Solid, dashed, dash-dotted lines correspond to $n_{B} / n_{0}=0,1.0,2.0$ respectively. $S_{0}\left(n_{B}\right)$ determined by the Borel stability method at each density is also shown in $\mathrm{GeV}^{2}$ unit. The Borel window is chosen to be $0.41 \mathrm{GeV}^{2}<$ $M^{2}<1.30 \mathrm{GeV}^{2}$.

Fig.2: A schematic illustration of the $V-N$ scattering with s-channel nucleon resonances.

Fig.3: (a) Borel curves for $m_{V}\left(M^{2}\right)$ at zero density using $\Pi_{\mu \mu}^{R}$ with several different values of $S_{0}$ in $\mathrm{GeV}^{2}$ unit. (b) Borel curves for $m_{V}\left(M^{2}\right)$ at nuclear matter density using $\Pi_{\mu \mu}^{R}$ with several different values of $S_{0}$ in $\mathrm{GeV}^{2}$ unit. 


\section{REFERENCES}

[1] G. E. Brown, Nucl. Phys. A 522 (1991) 397.

[2] Quark Matter '95, Nucl. Phys. A (1995).

[3] See the review, T. Hatsuda, Hadron Structure and the QCD Phase Transition, preprint hep-ph/9502345 (1995).

[4] T. Hatsuda and Su H. Lee, Phys. Rev. C46 (1992) R34.

[5] G. E. Brown and M. Rho, Phys. Rev. Lett. C66 (1991) 2720.

[6] K. Saito, T. Maruyama and K. Soutome, Phys. Rev. C40 (1989) 407;

H. Kurasawa and T. Suzuki, Prog. Theor. Phys. 84 (1990) 1030;

H.-C. Jean, J. Piekarewicz and A. G. Williams, Phys. Rev. C49 (1994) 1981;

H. Shiomi and T. Hatsuda, Phys. Lett. B334 (1994) 281.

[7] Y. Koike, Phys. Rev. C51 (1995) 1488.

[8] The seminal applications of the QSR to the nucleon in nuclear matter have been give in the following papers;

E. G. Drukarev and E. M. Levin, Prog. Part. Nucl. Phys. A556 (1991) 467.

T. Hatsuda, H. Hogaasen and M. Prakash, Phys. Rev. Lett. 66 (1991) 2851.

R. J. Furnstahl, D. K. Griegel and T. D. Cohen, Phys. Rev. Lett. 67 (1991) 961.

[9] R. J. Furnstahl and T. Hatsuda, Phys. Rev. Lett. 72 (1994) 3128.

R. J. Furnstahl, Phys. Rev. C50 (1994) 1735.

[10] T. Hatsuda, Y. Koike and S. H. Lee, Nucl. Phys. B394 (1993) 221.

[11] T. Hatsuda and T. Kunihiro, Nucl. Phys. B387 (1992) 715; Phys. Rep. 247 (1994) 221.

[12] M. Gluck, E. Reya and A. Vogt, Z. Phys. C48 (1990) 471.

[13] A. I. Bochkarev and M. E. Shaposhnikov, Nucl. Phys. B268 (1986) 220.

[14] N. V. Krasnikov, A. A. Pivovarov and N. N. Tavkhelidze, Z. Phys. C19 (1983) 301.

[15] A. Shifman, A. I. Vainshtein and V. I. Zakharov, Nucl. Phys. B147 (1979) 385.

[16] There are also $t$-channel meson exchanges in the process. One of them is the scalarmeson $(\sigma)$ exchange which gives energy independent contribution to the amplitude and is more relevent to the vector-meson mass shift in nuclear matter.

[17] Y. Kondo and O. Morimatsu, preprint INS-933 (1992) unpublished; Phys. Rev. Lett. 71 (1993) 2855.

[18] Particle Data Group, Phys. Rev. D50 (1994) 1173.

[19] For the readers who has not been convinced yet, we give here another argument. The FESR can be constructed by taking certain number of derivative of the real part of $\Pi_{\mu \nu}^{R}\left(Q^{2}\right)$ [14. If one start with $\Pi^{R}\left(Q^{2}\right) \sim \ln Q^{2}+a_{1} / Q^{2}+a^{2} / Q^{4}+a_{3} / Q^{6}$ and apply $Q^{2} \partial / \partial Q^{2}$, one obtains the canonical FESR (13). The local duality relation in (13) is nothing but the statement that $a_{1}=0$. On the other hand, If one start with $\Pi_{\mu \mu}^{R}\left(Q^{2}\right) \sim$ $Q^{2} \Pi^{R}\left(Q^{2}\right) \sim Q^{2} \ln Q^{2}+a_{1}+a^{2} / Q^{2}+a_{3} / Q^{3}$, any derivative with respect to $Q^{2}$ will loose the information of $a_{1}$. This is equivalent to the missing of the local duality relation or the ambiguity of $s \delta(s)$ in $\operatorname{Im}_{\mu \mu}^{R}(s)$. TH thanks Dr. K. Tanaka for discussion on this point.

[20] S. Choi, T. Hatsuda, Y. Koike and Su H. Lee, Phys. Lett. B312 (1993) 351.

[21] Su H. Lee, Phys. Rev. D49 (1994) 2242.

[22] M. Asakawa and C. M. Ko, Phys. Rev. C48 (1993) 526.

[23] C.M.Ko P. Levai, X.J. Qiu and C.T.Li, Phys. Rev. C45 (1992) 1400. 


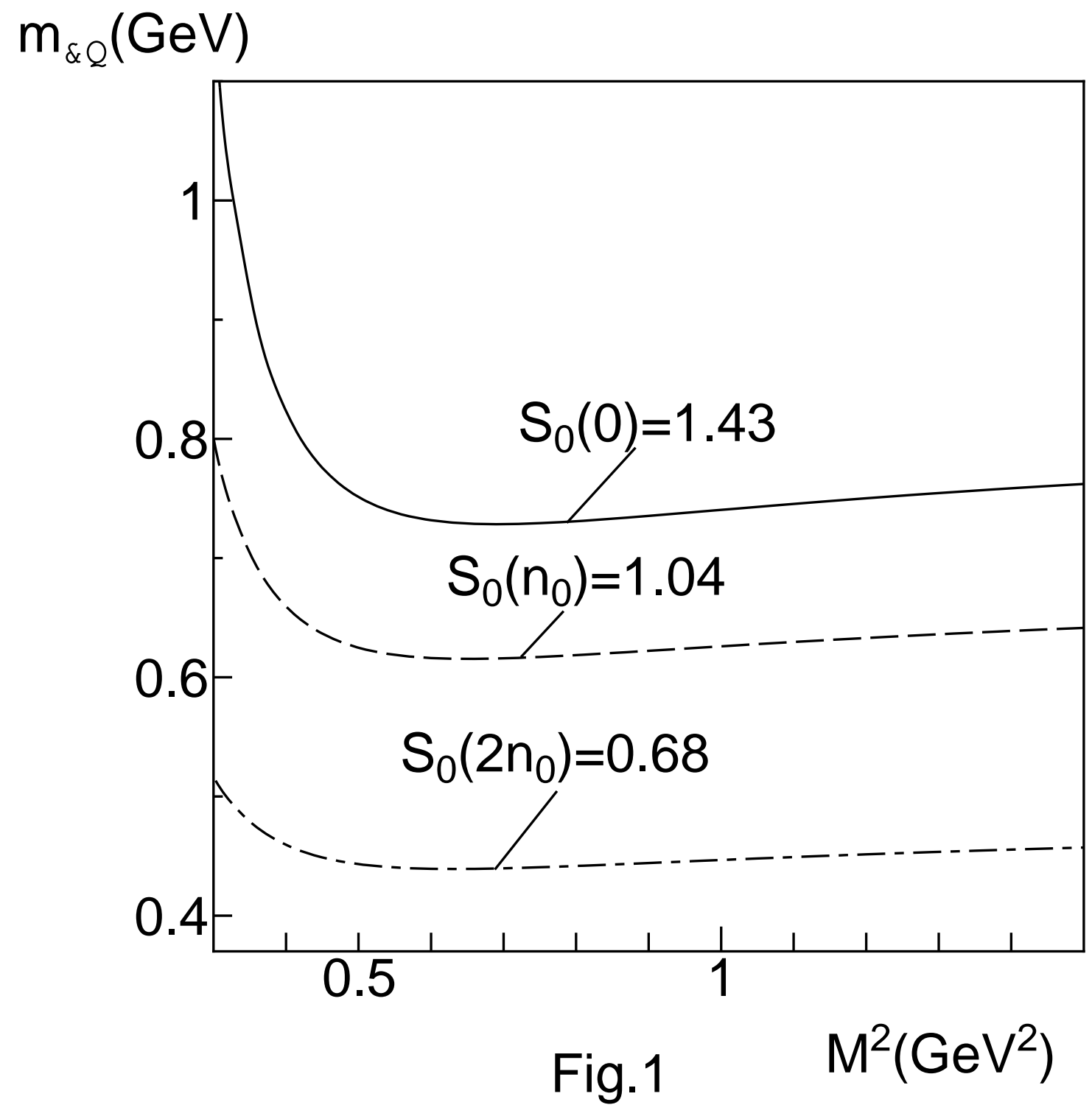




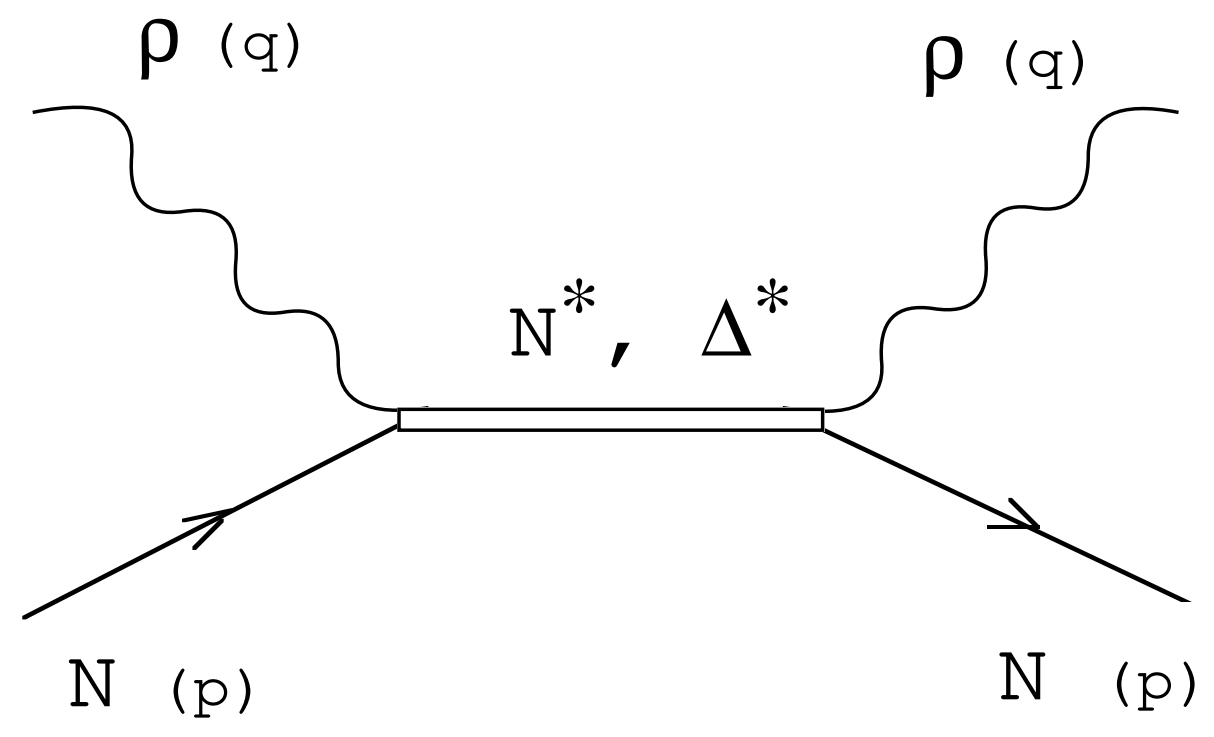

Fig. 2 


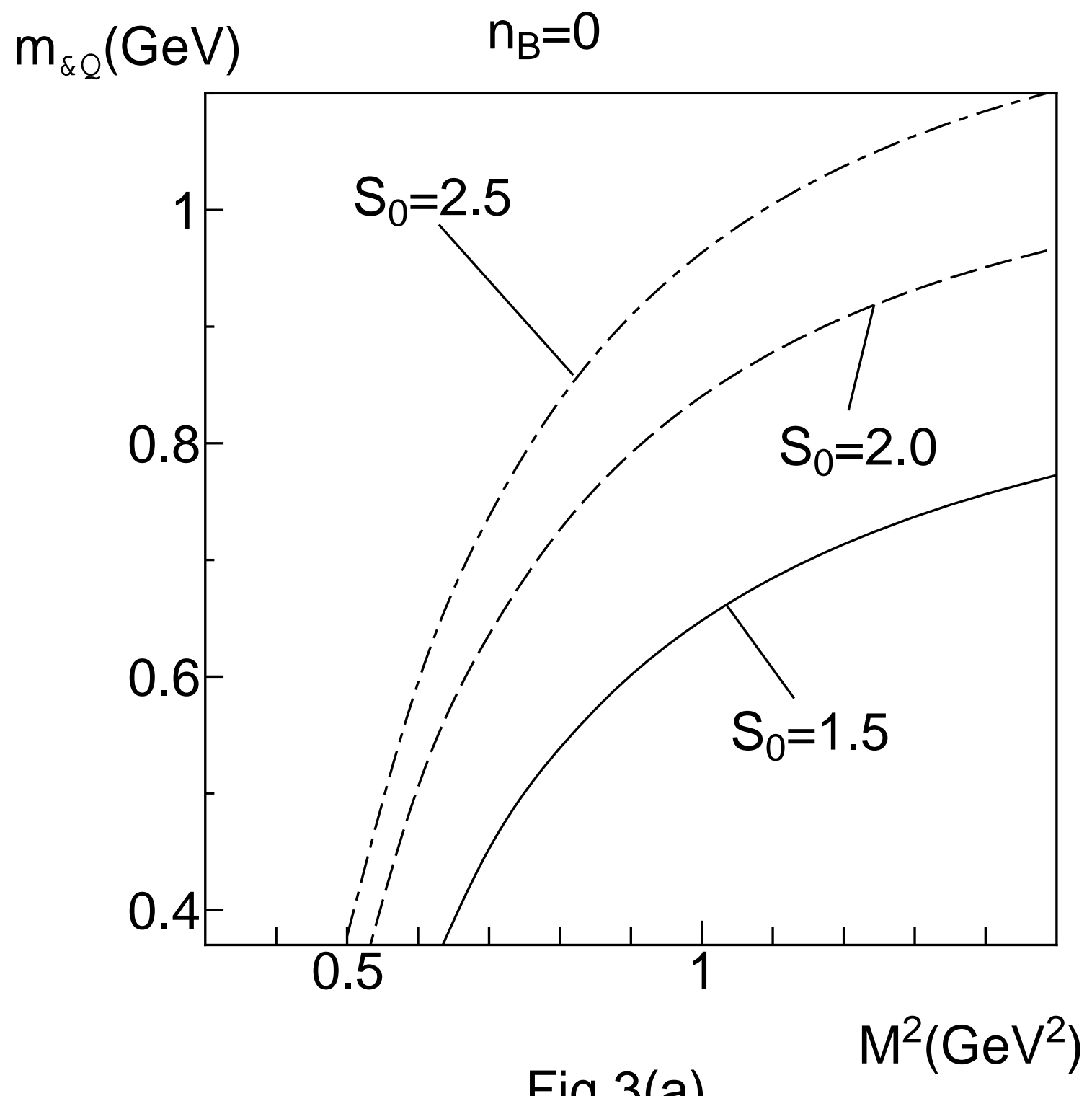

Fig.3(a) 


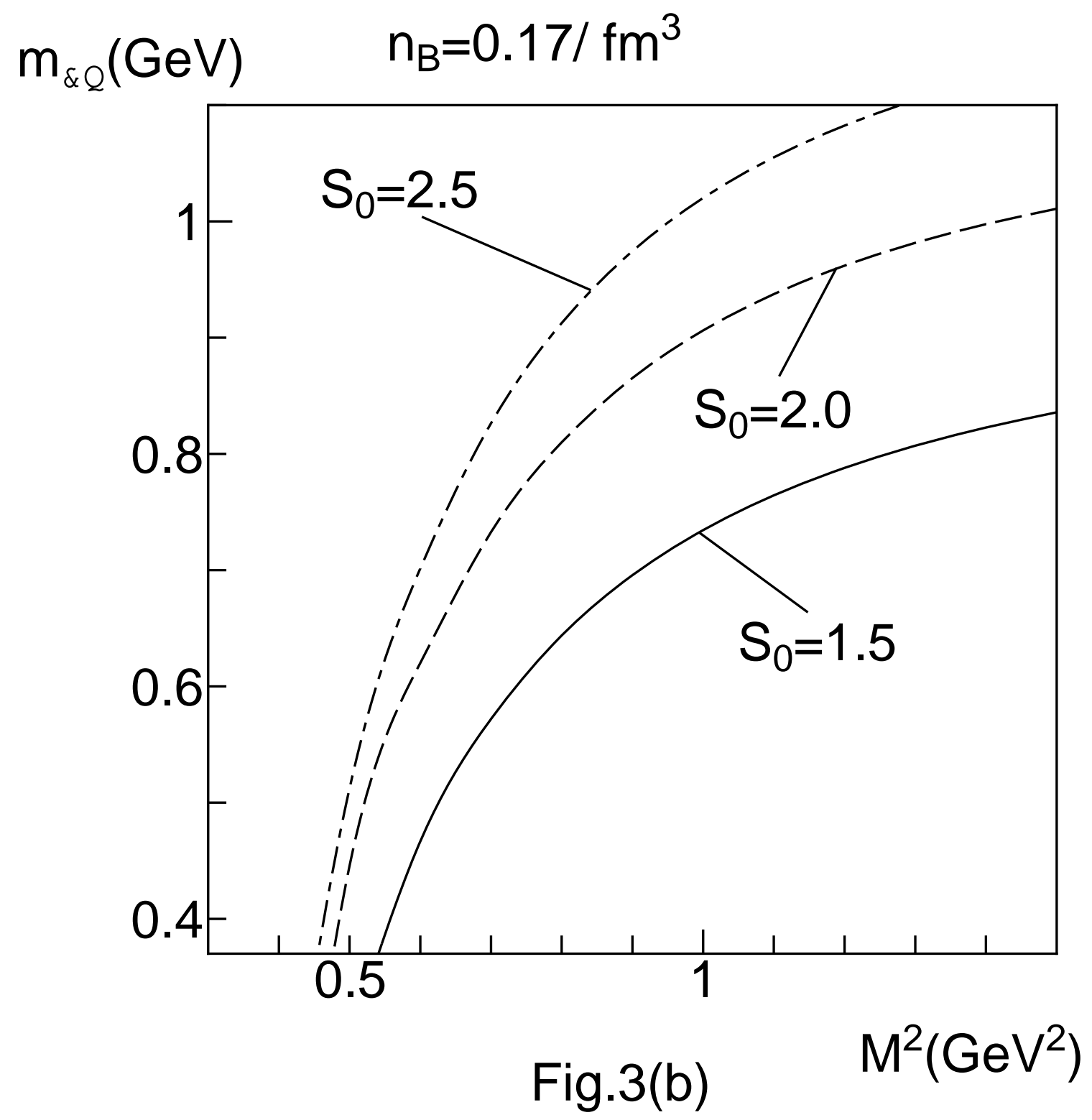

